\title{
Polylogarithmic Store-Carry-Forward Routing using Mobile Nodes
}

\author{
Jie $\mathrm{Wu}$ and Shuhui Yang \\ Department of Computer Science and Engineering \\ Florida Atlantic University \\ Boca Raton, FL 33431 \\ E-mail: jie@cse.fau.edu, syang1@fau.edu.
}

\begin{abstract}
It is well known that mobility increases the capacity of mobile ad hoc networks (MANETs) by reducing the number of relays for routing, prolonging the lifespan of wireless sensor networks (WSNs) by using mobile nodes in place of bottleneck static sensors, and ensuring network connectivity in delay-tolerant networks (DTNs) using mobile nodes to connect different parts of a disconnected network. Trajectory planning and the coordination of mobile nodes are two important design issues aiming to optimize or balance several measures, including delay, average number of relays, and moving distance. In this paper, we propose a new controlled mobility model with an expected polylogarithmic number of relays to achieve a good balance among several contradictory goals, including delay, the number of relays, and moving distance. The model is based on the small-world model where each static node has "short" link connections to its nearest neighbors and "long" link connections to other nodes following a certain probability distribution. Short links are regular wireless connections whereas long links are implemented using mobile nodes. Various issues are considered, including trade-offs between delay and average number of relays, selection of the number of mobile nodes, and selection of the number of long links. The effectiveness of the proposed model is evaluated analytically as well as through simulation.
\end{abstract}

Keywords: Delay tolerant network (DTN), mobile ad hoc network (MANET), routing, simulation, small-world model, wireless sensor network (WSN).

\section{INTRODUCTION}

The traditional connection-based model (such as TCP/IP) used in MANETs and WSNs is built on the premise that the underlying network is connected and views node mobility as undesirable. However, mobility is treated as a side issue through a simple recovery scheme. For example, a route disruption caused by node movement is restored by either route rediscovery or a local fix in a typical reactive approach, assuming either that node movement is infrequent or that a node moves relatively slowly with respect to its transmission range. More recently, mobility has been identified as a serious threat to the traditional model [17]. The threat is mainly

1-4244-1455-5/07/\$25.00 @ 2007 IEEE caused by the asynchronous sampling of Hello messages and various protocol delays that result in an inconsistent global state.

The more recent movement-assisted model tries to exploit node mobility for the routing process. The movement-assisted model typically follows a store-carryforward paradigm, where a mobile node first stores the routing message, carries it while moving randomly or on a controlled path, and then forwards it to either an intermediate node or the destination. This model is motivated by the following potential application areas: (1) In MANETs, the network capacity increases with resourcerich (in terms of processing, memory, and energy) mobile nodes to reduce the average number of relays in a routing process [6]. (2) In WSNs, the network lifetime is prolonged with mobile nodes in place of (bottleneck) static sensor nodes [14], [8] to support relay and/or simple processing. (3) In DTNs, the network connectivity can be ensured using mobile nodes to connect various parts of a disconnected network [20].

Movement-assisted models can be classified based on random (uncontrolled) movement (epidemic routing [13]) and controlled movement ([20]). In controlled movement, various design issues exist, including the number of mobile nodes, trajectory planning, and node communication and synchronization. Although extensive work has been conducted on both models, relatively little work has been done on controlling the amount of relays in a routing process. In the traditional model, the average number of relays grows with the spatial diameter of the network (diameter-hop-count), that is, $O(\sqrt{n})$, where $n$ is the number of nodes, assuming some sort of topology control has been applied to reduce the network density for energy saving and collision reduction. At the other end of the spectrum, most controlled, movement-assisted schemes use a constant number of relays. However, these schemes incur high latency issues in packet forwarding.

Inspired by the small-world model of Watts and Strogatz [15], we consider a random, movement-assisted scheme to achieve a moderate constraint, which is an expected number of relays equal to $(\log m)^{2}$ in an $m \times m$ 
square area. The corresponding method is simply called polylogarithmic store-carry-forward. The main objective of using such a moderate constraint is to obtain better performance in other metrics, including latency, while maintaining moderate end-to-end throughput of each node, which is $O\left(\frac{1}{\log m}\right)$, based on the analysis of the connection-based model by Gupta and Kumar [7]. Specifically, in a small-world model, each static node has "short" link connections to its nearest neighbors and "long" link connections to other nodes following a certain probability distribution. Each short link is a regular wireless connection whereas each long link is implemented using a mobile node with a moving trajectory that follows the long link. When long links follow a certain distribution, nodes can construct short paths effectively using local information. Here, a path consists of both short and long links. The trajectory of mobile nodes, however, is not limited to long links. It can be extended to include short links and to share multiple long links.

Although the small-world model has been used in other fields, such as searching in the unstructured peerto-peer networks, no systematic study has been done on its applications in MANETs, WSNs, or DTNs [5] using mobile nodes to emulate long links. In this paper, we will address some unique challenges in implementing the small-world model in emerging networks with mobile nodes, discuss some design issues related to parameter selections, and weigh several interesting trade-offs among delay, average number of relays, and moving distance. More specifically,

1) we propose a polylogarithmic store-carry-forward model based on the small-world model,

2) we present an in-depth analysis on the use of mobile nodes to emulate long links (remote contacts) in the small-world model,

3) we devise both static and dynamic trajectory planning of mobile nodes for improving routing performance and reliability,

4) we discuss various design issues, and

5) we conduct extensive simulation on a custom simulator to validate the effectiveness of the proposed model.

The following assumptions are used in this paper. (1) Wireless nodes are either static or controlled mobile nodes. Mobile nodes are resource rich, such as a vehicle in a vehicle ad hoc network (VANET), compared with static nodes, and have no memory capacity limit. (2) Two static/dymanic nodes are neighbors if they are within the transmission range, which is set to one unit in this paper. (3) Each node (including the source) knows its location and the location of the destination. This can be achieved through GPS or non-GPS localization methods. In particular, when destination refers to a particular node (rather than a geographical location), some form of location management will be used, such as home region [16]. (4) Mobile nodes move with constant velocity. This model can also be easily extended to incorporate mobile nodes with variable velocities. (5) Data exchange between two nodes, static or dynamic within each other's transmission range, can be done instantly.

\section{RELATED WORK}

\section{A. Mobility in MANETs, WSNs, DTNs, and VENETs}

Grossglauser and Tse show that mobility increases the capacity of MANETs based on Gupta and Kumar's model on network capability [7]. A series of efforts have been made based on the store-carry-forward paradigm, where a mobile node first stores the routing message, carries it for a while moving either randomly or in a controlled manner, and then forwards it to an intermediate node or the destination. Epidemic routing [13] uses a random mobility model together with packet replication to speed up the delivery process.

In WSNs, most models assume that sensor nodes are static. More recent work considers a type of resource rich mobile sensor for sensor coverage and lifetime extensions. The idea of using mobile sensors for sensor coverage enables the re-deployment of some mobile sensors to undercovered areas [8], [14]. The idea of lifetime extension enables the use of resource rich mobile sensors to emulate the function of bottleneck or energy-depleting nodes, which can be a relay node or a sink node. When a mobile node is used to emulate a sink node, the sink can stay in multiple positions.

Routing in DTNs can be classified as random movement or controlled movement. The work in [11] also includes algorithms for data delivery in disconnected networks using node mobility. In message ferrying (MF) [20], some ferries, which are nodes that have completely predictable routes through the geographic areas, are employed for data delivery. Nodes route packets end-to-end using the ferries. Ferries move around according to the known routes.

The VANET [5], [19] is a form of mobile ad hoc network, to provide communications among nearby vehicles and between vehicles and nearby fixed equipment. Rather than moving at random, vehicles tend to move in an organized fashion. Most vehicles are power-rich and restricted in their range of motion, for example by being constrained to follow a paved highway. Data routing is also conducted in a store-carry-forward way. The vehicle routing problem (VRP) [2] is a generalization of the traveling saleperson problem (TSP), which considers scheduling vehicles to support customers with known demands, minimizing the total distance travelled [4]. 


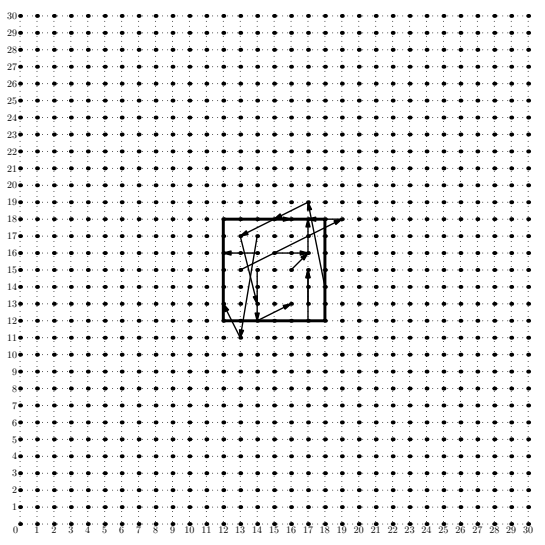

(a) Center area $6 \times 6$ of a $30 \times 30$ network

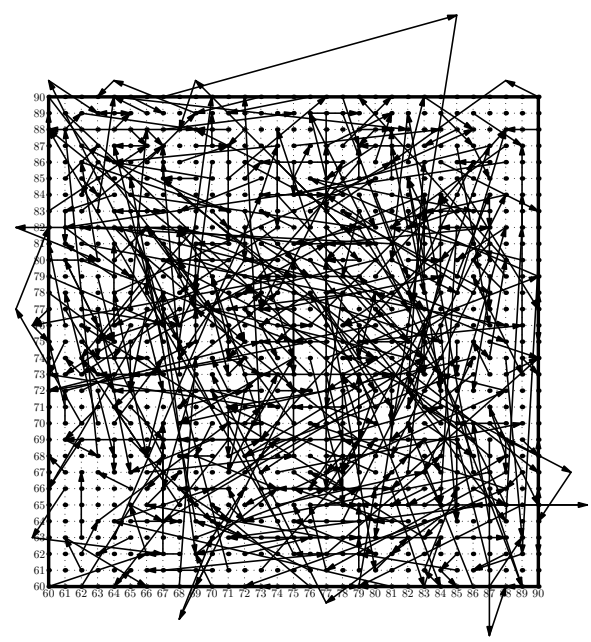

(b) Center area $30 \times 30$ of a $150 \times 150$ network

Fig. 1. Sample small-world networks.

\section{B. Small-world models}

Kleinberg [9] was the first to consider the smallworld phenomenon from the algorithmic perspective, and proposed a model of a "navigable" small-world network that can find short paths between any two points in the network using local information only. Specifically, a 2$\mathrm{D}$ grid model in an $m \times m$ space is considered, where each point has four local contacts (links) to its nearest neighbors and $q$ long-range contacts (long links). The existence of a long link from $u$ to $v$ has a probability proportional to $d(u, v)^{-r}$, where $d(u, v)$ is the distance between them and $r$ is a constant. It is shown that when $r=2$, the expected delivery time is $O\left((\log m)^{2}\right)$ (called polylogrithmic in terms of $m$ ). The extension to a navigable hierarchical network is discussed in [10].

The small-world model has been successfully applied to support efficient searching in unstructured peer-to-peer networks. However, the extensions to wireless networks have been limited due to the lack of efficient means to support long links, although the potential advantage of using the small-world model was pointed out back in 2001 [1]. The existing applications implement long links using wireline links [3] or at the application layer [12]. The former approach relies on wireline connections. In addition, the long link does not follow the smallworld distribution. The latter case shifts the burden to the application layer. In our approach, we adopt a slightly different model for long link distributions. Long links are implemented by resource rich mobile nodes.

\section{POLYLOGRITHMiC STORE-CARRY-ForWARD}

\section{A. Basic model}

We assume a grid-based model [18] in an $m \times m$ space with the horizontal and vertical dimensions. Each $1 \times$
1 grid is associated with an address $(i, j)$ where $i, j \in$ $0,1,2, \ldots, m$. All the deployed sensor nodes in a grid form a cluster and a clusterhead is selected to deal with the inter-grid communication. We simply view the cluster as a single node that has the address of its grid. We define the Manhattan distance between two nodes $u=(i, j)$ and $v=\left(i, j^{\prime}\right)$ to be the number of horizontal and vertical steps separating them:

$$
d(u, v)=\left|i-i^{\prime}\right|+\left|j-j^{\prime}\right| .
$$

Each node $u=(i, j)$ has four local links connecting four neighbors: $(i-1, j),(i, j-1),(i+1, j)$, and $(i, j+$ $1)$, using a unit uniform wireless transmission range. In addition, $u$ has $q(\geq 1)$ long links. The probability of a long link to $v$ such that $d(u, v) \leq 2 m$ is $c[d(u, v)]^{-2}$ and the probability of a long link to $v$ such that $d(u, v)>2 m$ is 0 . Constant $c$ should be selected so that

$$
\sum_{v} c[d(u, v)]^{-2}=q
$$

To avoid boundary conditions, the $m \times m$ space is situated at the center of a $5 m \times 5 m$ space and nodes outside $m \times m$ can be used to relay, but not as sources or destinations. Although nodes not in the original $m \times m$ have contacts (through long links) outside the $5 m \times 5 m$ region, they will not be used to assist the routing process. Figure 1 (a) shows a sample $6 \times 6$ network together with its $30 \times 30$ expanded network. Note that all contacts in the $6 \times 6$ network are within the $30 \times 30$ network. All contacts outside the extended network are not shown. Note that the proposed model is slightly different than the one in [9] in that we allow nodes in the $m \times m$ space to have long link contacts to the outside of the area. We can see that not all of the nodes in the central $m \times m$ and the extended $5 m \times 5 m$ regions have valid long links (and these links are 
not shown). This is because their long links are directed to the nodes in the expanded area which are far away from the central network. If we still use them as relays, the detour will be too large. However, if the relays do not generate too much additional moving distance, as will be discussed later in the long link jump condition, we can still use them. Figure 1 (b) is a sample in a larger scaled network, where the center $30 \times 30$ area of a $150 \times 150$ network, is shown.

In the basic model, it is assumed that there are sufficient numbers of static and controlled mobile nodes so that there is at least one static node and one dynamic node in each grid point. At each grid point, one static node is used to act as a place holder. This node generates a new packet intended for a destination, forwards, or stores a bypass packet. The mobile node circulates around the long link $(u, v)$. This node picks up one or more packets at $u$ and delivers them to $v$. We will discuss various solutions when the density condition fails. These solutions include extending the size of each grid point and the use of one mobile node to emulate functions of multiple static and dynamic nodes at several grid points.

The routing algorithm, inspired by the one in [9], follows a greedy approach where at each step, the current place holder $u$ chooses a contact, through a short or long link, that is as close to the destination $t$ as possible. The short link is through regular wireless communication while the long link is through a mobile node moving between $u=(i, j)$ and $v=\left(i^{\prime}, j^{\prime}\right)$. Typically, an $X-Y$ routing in a 2-D mesh can be used, where the mobile node circulates along the circular trajectory $(i, j) \rightarrow\left(i^{\prime}, j\right) \rightarrow$ $\left(i^{\prime}, j^{\prime}\right) \rightarrow\left(i, j^{\prime}\right) \rightarrow(i, j)$

More specifically, the algorithm operates in phases. It is in phase $i$ if the current node $u$ satisfies $2^{i}<d(u, t) \leq$ $2^{i+1}$. At node $u$ in phase $i$, a long link $(u, v)$ is used if $d(v, t) \leq 2^{i}$. This condition is simply called the long link jump condition (Figure 2). Kleinberg [9] showed that in phase $i$, the expected time before the current place holder has a long link contact within Manhattan distance $2^{i}$ of $t$ is bounded proportionally to $\log m$. Since there are $\lceil\log 2 m\rceil$ phases, from phase 0 to phase $\lceil\log 2 m\rceil-1$, a bound on the number of links is proportional to $(\log m)^{2}$ (called polylogrithmic store-carry-forward) follows.

\section{B. Basic properties}

Theorem 1: The probability of a long link $(u, v)$ that satisfies the long link jump condition is at least $\frac{1}{2^{6}} H(2 m)^{-1}$, where $H(2 m)$ is the Harmonic series, which is defined as $H(n)=\sum_{i=1}^{n} \frac{1}{i}$. In addition, $\ln (n+1) \leq H(n) \leq \ln (n)+1$. So $H(n)$ is very close to $\ln (n)$ for a large $n$.

Proof: Suppose node $u$ has a packet intended for

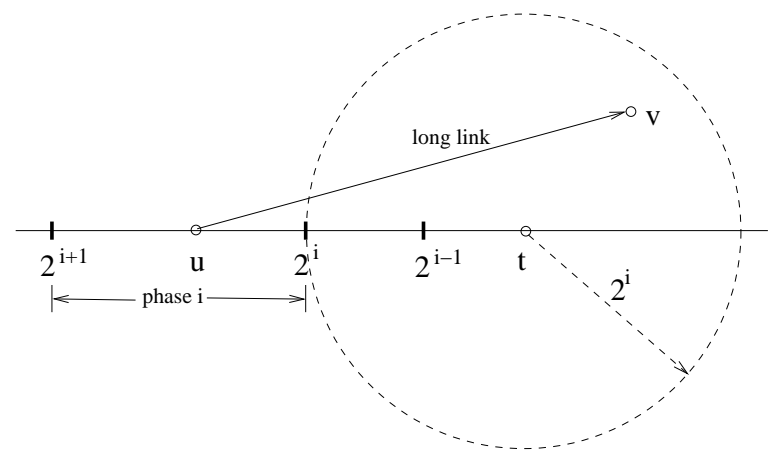

Fig. 2. The greedy routing approach in phases and the long link jump condition.

destination $t$. The probability that $u$ chooses $v$ is

$$
d(u, v)^{-2} / \sum_{v \neq u} d(u, v)^{-2},
$$

where $\sum_{v \neq u} d(u, v)^{-2}=\sum_{i=1}^{2 m}(4 i)\left(i^{-2}\right)=4 \sum_{i=1}^{2 m}(i)^{-1}$ $=4 H(2 m)$. Hence, the probability that $v$ is chosen is

$$
\left[4 H(2 m) d(u, v)^{2}\right]^{-1} .
$$

Assume $S_{i}$ is the set of nodes within distance $2^{i}$ of $t$ reachable from $u$. When phase $i=\lceil\log 2 m\rceil-1$,

$$
\left|S_{i}\right| \geq 1+\sum_{j=1}^{2^{i}} j>2^{2 i-1} .
$$

Each element in $S_{i}$ is within distance $2^{i+1}$ of $u$ since the length of a long link is bounded by $2 \mathrm{~m}$. Thus, the message enters $S_{i}$ with a probability of at least

$$
\frac{2^{2 i-1}}{4 H(2 m) 2^{2 i+2}}=\frac{1}{2^{6}} H(2 m)^{-1} \text {. }
$$

When $i<\lceil\log 2 m\rceil-1,\left|S_{i}\right|=1+4 \sum_{j=1}^{2^{i}} j=$ $2\left(2^{i}\right)^{2}+2\left(2^{i}\right)+1>2^{2 i+1}$. Here, each element in $S_{i}$ is within distance $2^{i+1}+2^{i}<2^{i+2}$. Thus the message enters $S_{i}$ with a probability of at least

$$
\frac{2^{2 i+1}}{4 H(2 m) 2^{2 i+4}}=\frac{1}{2^{5}} H(2 m)^{-1} \text {. }
$$

Hence, the probability of a long link $(u, v)$ that satisfies the long link jump condition is at least $\frac{1}{2^{6}} H(2 m)^{-1}$.

Note that when compared with the result in [9], since this result has the same asymptotic result, all other results in [9] stay. For example, the average number of short links at each phase is the reverse of the long link probability, i.e., $O(H(2 m))$ which is $O(\ln m)$, and hence, $O(\log m)$. Since there are $\lceil\log 2 m\rceil$ phases, the expected total moving distance through short links is $O\left((\log m)^{2}\right)$. The following result shows the worst case moving distance for both short and long links. 
Theorem 2: The total moving distance between source $s$ and destination $t$ through short links is bounded by $d(s, t)$ and the total moving distance through long links is bounded by $5 d(s, t)$.

Proof: Suppose $s$ is in the $k$ th phase (with respect to $t)$. There are $k+1$ phases, $0,1,2, \ldots, k$. In phase $k$, the maximum number of short links is bounded by $d(s, t)-$ $2^{k}$. In phase $i$, with $0 \leq i<k$, the maximum number of short link moves is $2^{i+1}-2^{i}$. If there is a long link jump at $u$ in phase $i$, the new contact $v$ is in phase $i-1$ or less. Once phase 0 completes, the current node is either the destination (through a long link jump) or a node that is a neighbor of the destination, and hence, one more short link is needed. Therefore, the total moving distance through short links is bounded by

$$
\left(d(s, t)-2^{k}\right)+\left(\sum_{i=0}^{k-1} 2^{i+1}-2^{i}\right)+1 \leq d(s, t) .
$$

In phase $k$, the distance of the long link is bounded by $d(s, t)+2^{k}$ based on the triangle inequity. In all other phases $i$, with $0 \leq i<k$, the distance of the long link jump is bounded by $2^{i+1}+2^{i}$ based on the triangle inequity, since $d(u, t) \leq 2^{i+1}$ and $d(v, t) \leq 2^{i}$. Therefore, the total moving distance through long links is bounded by

$$
\left(d(s, t)+2^{k}\right)+\left(\sum_{i=0}^{k-1} 2^{i+1}+2^{i}\right)<d(s, t)+4\left(2^{k}\right)<5 d(s, t) .
$$

\section{Design issues}

In this paper, we will focus on several unique issues when mobile nodes are used to emulate long links in an emerging network with mobile nodes.

In a network with mobile nodes, any intermediate nodes on the trajectory of a long link $(u, v)$ can attach their packets to the mobile node if $v$ is closer to their intended destination. These intermediate nodes are freeriders. Trajectory sharing refers to sharing of multiple long links that form a cycle to reduce waiting delay. The effect of multiple long links includes the average path length in terms of delay, average number of relays, and moving distance. Delay, average number of relays, and moving distance trade-offs deal with trading between long links, which have relatively long delay (and moving distance) but fewer number of relays, and short links, which have a greater number of relays but short delay (moving distance).

\section{Design Details}

\section{A. Free-riders}

In the small-world model, each long link $(u, v)$ is directional, where $u$ is called home and $v$ the destination.
The mobile node circles along $(u, v)$ first to $v$ and $(v, u)$ back to $u$. Two methods of circulation can be used: (1) home-based, where the mobile node stays at node $u$ unless there is a packet intended for $v$; (2) movementbased, where the mobile node keeps on circulating.

There are two types of free-riders (also called data hitchhikers) for a long link $(u, v)$. (1) Node $u$ has a packet for destination $t$ and $(u, w)$ for an intermediate node $w$ meets the long link jump condition. Node $w$ is called a type I free-rider for link $(u, v)$ (see Figure 3 (a)). (2) Intermediate node $w$ on the trajectory has a packet for destination $t$ and $(w, v)$ meets the long link jump condition. Node $w$ is called a type II free-rider for link $(u, v)$ (see Figure $3(\mathrm{~b})$ ).

In the movement-based approach, we assume the mobile node will stay at each intermediate node for a short period of time $\Delta$ to pick any free-riders. The following results show the probability of free-riders given the probability $p$ of a long link $(u, v)$.

Theorem 3: For a given packet at $u$, the probability for the existence of a type I free-rider is $O(1) p$. The probability of a type II free-rider for each intermediate node on the trajectory to destination $v$ is also $O(1) p$.

The proof of the theorem is omitted due to the space limit. A more general type of free-rider is from $w_{1}$ and $w_{2}$ when both of them are on the trajectory of a mobile node on the long link $(u, v)$ (including the return path $(v, u)$ ) that satisfies the long link jump condition with respect to a destination $t$. In this case, we pose an additional constraint on the trajectory from $w_{1}$ to $w_{2}$ to meet the triangle inequity property as shown in Theorem 2 . Another stricter constraint is to ensure a shortest path from $w_{1}$ to $w_{2}$, i.e., the trajectory on the cycle corresponds to the shortest path from $w_{1}$ to $w_{2}$. The shortest path condition can be ensured by allowing at most one turn (X dimension to $\mathrm{Y}$ dimension or $\mathrm{X}$ to $\mathrm{Y}$ ) in the routing process as shown in Figure 3 (c). Both Theorems 3 holds for the general type of free-rider. When the source node $w_{1}$ tries to find its long link $\left(w_{1}, w_{2}\right)$ in the trajectory of $(u, v)$ (including $(v, u))$, it should check every node from $w_{1}$ to a reachable node in the trajectory with at most one turn. There may be more than one node that satisfies the long link jump condition. In this case, the one that is closest to $t$ is selected as $w_{2}$.

\section{B. Long and short link trade-offs}

Long and short link trade-offs deal with delay, average number of relays, and moving distance. These trade-offs are important in networks like MANETs and WSNs, since the average number of relays directly relates to network capacity in MANETs and energy consumption of static sensors. Although the expected number of short links is $H(2 m)$ before using a long link at each phase, a long link 


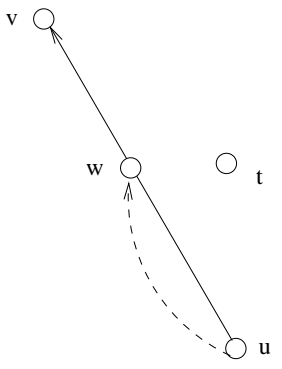

(a)

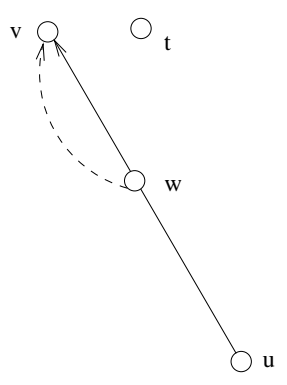

(b)

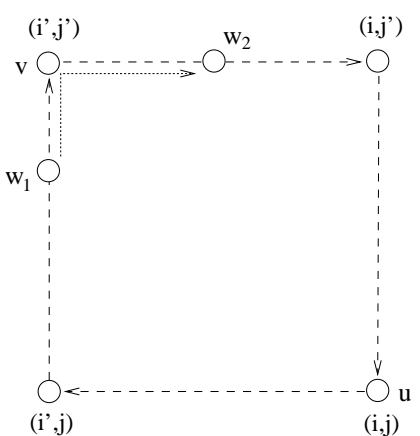

(c)

Fig. 3. Free-riders: (a) type I, (b) type II, and (c) general type with one turn between $w_{1}$ and $w_{2}$.

is skipped for short links in the following situations: (1) To avoid regular moving delay. The speed of a moving node is significantly lower than that of a normal wireless transmission through a short link. (2) To avoid waiting delay. The moving node may not be available when a packet is ready to be forwarded at an intermediate node. (3) To avoid excessive moving delay. Excessive moving delay occurs when several long links in a cycle have to be used before reaching the next contact.

The local place holder needs to keep some information to facilitate the above trade-offs. The regular moving delay can be calculated by dividing the length of each long link by the moving speed of mobile nodes. A counter is needed for each link to keep track of the remaining time of the round trip of a mobile node along the long link. The trading point is the threshold above which short links are used to trade long links.

Excessive moving delay can be avoided or reduced without resorting to local links. For example, if the objective is to limit the number of long link relays, then a node $u$ in phase $i$ is allowed to use the long link contact $v$ separated by $k$ long links in a cycle if $v$ is within $2^{i-k}$ of destination $t$. To avoid excessive moving distance, a shortcut can be used to reach $v$ directly. However, $k-1$ intermediate nodes are skipped and packets initiated from these nodes cannot be forwarded. This situation can be mended by using multiple mobile nodes with some following the regular trajectory and others following the shortcut if needed.

\section{Multiple long links}

When $q>1$, multiple long links per grid point are available. We assume that long links are independent and follow the same distribution model. Multiple long links provide more choices. However, they do not give a linear speedup in terms of path length reduction. When $k$ is small, the probability of area overlap is small, which corresponds to a close-to-linear speedup in path reduction. As $k$ increases, the probability of area overlap increases, and linear speedup cannot be maintained although path reduction continues.

Multiple long links can increase the probability of cycle formation among them to reduce delay. However, the cycle formation process is more complex since each node has multiple outgoing links and probably multiple incoming links. A node may be involved in multiple cycles. These multiple cycles can themselves be combined to form large cycles. Based on the delay reduction theorem using cycles, the reduction rate is a constant (two) which is independent of the size of the cycle. Therefore, it is preferable that a short cycle is maintained for the purpose of reliability (multiple cycles to guard against broken cycles) and memory efficiency (shortened route information stored at each node of a cycle).

To find a short cycle, the Dijkstra's shortest path algorithm can be applied. The following approach can be used with local information only: To avoid generating large cycles, each node performs an expanded ring search for a cycle using TTL by sending out an "initiation message". The TTL is incremented linearly or exponentially at each round. A node $u$ stops sending out "initiation message" if a cycle is found and $u$ is the initiator. However, node $u$ still participates in the forwarding process of "initiation message" packets. In addition, nodes other than the initiator in the cycle still send out "initiation message". The initiation process at a particular node stops when either a cycle is found or TTL exceeds a predefined threshold.

\section{Reactive long link initialization}

In order to improve the ratio of the usage of long links, we developed the reactive long link initialization approach. We call the basic PSCF a proactive PSCF where the trajectory of each mobile node is determined before the execution of the system. Then, in the reactive PSCF, the long link of each place holder is calculated during the initialization phase, but its direction changes to the direction of the destination of the first generated packet in its corresponding place holder. 
In the small-world model, for a fixed node $u$, the existence of a long link from $u$ to any other fixed node $v$ in the network has a probability proportional to $d(u, v)^{-r}$. Therefore, generally speaking, the probabilities of the long link for node $u$ pointing to each direction are identical, i.e. the direction is randomized. In the reactive PSCF, although the direction of the long link changes, it is still randomized. This is because the destination of the first packet is generated randomly. Therefore, the network is still a small-world model.

In the reactive $\mathrm{PSCF}$, since the direction of the long link is determined based on the direction of the destination of the first generated packet, the probability of the packet being delivered via the long link is increased. The performance of PSCF will increase, too. However, none of the subsequent generated packets take any advantage of the reactive long link initialization. In the long run, we can reset the direction of the long link according to the first packet in the waiting list when the mobile node returns each time. Thus, the overall performance can be improved, especially when the data rate is relatively low.

\section{E. Trajectory sharing}

Using mobile nodes for long links causes delay, the expected delay for a long link $(u, v)$ is $d(u, v) / v(m)$, where $v(m)$ is the moving velocity of the mobile node. We assume $\Delta$ time period at each intermediate node is included in the calculation of $d(u, v) / v(m)$. Trajectory sharing deals with multiple long links forming a cycle with multiple mobile nodes circulating around it. Specifically, suppose $\left(u_{1}, u_{2}\right),\left(u_{2}, u_{3}\right), \ldots,\left(u_{k}, u_{1}\right)$ forms a cycle and $d\left(u_{i}, u_{i+1}\right)=l_{i}$ and $d\left(u_{k}, u_{1}\right)=l_{k}$. We have $k$ mobile nodes circulating around the cycle of length $\sum_{i=1}^{k} l_{i}$.

Theorem 4: The expected average delay using trajectory sharing reduces the expected delay by half.

The proof of the theorem is omitted due to the space limit. Note that the expected average delay is for all nodes in the cycle. Each individual node may have an increased expected delay. For example, suppose three nodes in a cycle have link lengths $l_{1}=1, l_{2}=5$, and $l_{3}=6$. The expected average delay is 2 , which is larger than the individual delay of $l_{1}$.

Cycle structures also offer "look ahead" capability. Let us consider node $u_{1}$ in cycle $\left(u_{1}, u_{2}\right),\left(u_{2}, u_{3}\right)$, $\ldots,\left(u_{j}, u_{j+1}\right), \ldots,\left(u_{k}, u_{1}\right)$. Instead of using $u_{2}$ as the only possible long link jump, $u_{1}$ can use any other node $u_{2}, \ldots, u_{j}, \ldots, u_{k}$ for its long link contact. The link selection criterion still stands; that is, if $u_{1}$ is in phase $i$ with respect to destination $t$, the selected long link contact $u_{j}$ should be within $2^{i}$ of $t$. An additional constraint can be placed on the trajectory from $u_{1}$ to $u_{j}$, to meet the triangle inequity property as shown in Theorem 2 , that is,

$$
\sum_{i=1}^{j-1} l_{i} \leq d(u, t)+d\left(u_{j}, t\right),
$$

or an even stricter condition can be imposed, say, all intermediate nodes $u_{2}, u_{3}, \ldots, u_{j-1}$ should be along the shortest path from $u_{1}$ to $u_{j}$.

To detect cycles when $q=1$, each node initiates an "initiation" message and sends it to its long link neighbor unless an "initiation" is received. The initiation time is randomly selected at each node for a given period to reduce simultaneous initiations. When node $u$ receives an "initiation" message, it performs one of the following actions: (1) If it has not sent out its initiation, $u$ will forward the initiation to its neighbor (along the long link) after attaching its ID in the route field. (2) If it has sent out its initiation, the current message will be dropped. (3) If the current initiation contains the node ID of $v$, the complete route is copied at $u$, the message is changed to "found" with $v$ as the initiator and is then forwarded to its neighbor. When node $u$ receives a "found" message, a copy of the complete route is made unless $u$ is the initiator of the "found" message. In the latter case, the message is dropped.

\section{F. Sparse mode}

The proposed model and the corresponding PSCF can be extended to the sparse mode, where some place holders may not have mobile nodes to associate with them. We can set the density of mobile nodes, $r$, to control the sparse degree. This also means that each place holder has the probability $r$ to have a mobile node that belongs to it. The distribution of mobile nodes is random.

In the sparse mode, the trajectory of mobile nodes is important in networks such as DTNs and VENETs to connect parts in a disconnected network (consisting of static nodes). Mobile node shortages can be remedied through sharing, such as using fewer mobile nodes in a cycle and one mobile node to route around multiple long links from the same node.

In the sparse mode, each place holder still generates packets according to the data rate. If no mobile node is available, the packets stay at the place holder until there are some bypassing mobile nodes. The bypassing mobile nodes can serve as long links for the current place holder if the long link jump condition is met. Or, they can serve as short links to carry the packets to the four neighbors of the current place holder. Therefore, the degree of connectivity of the original partitioned network can be increased using node mobility.

\section{Simulation}

\section{A. Simulation environment and settings}

We set up the simulation in a $m \times m$ square area. Each $1 \times 1$ grid (viewed as a single point/node) is associated 


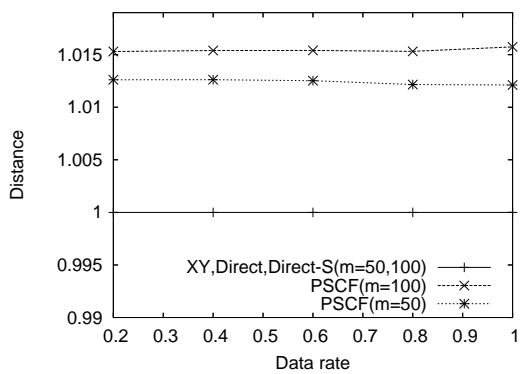

(a) Distance

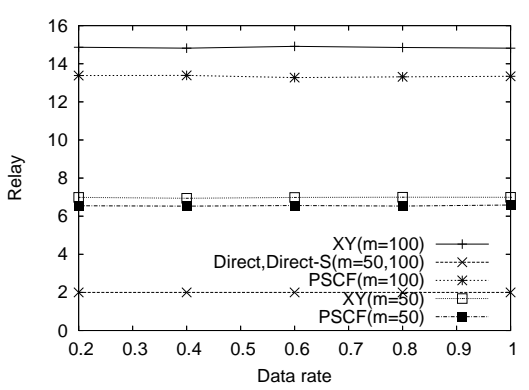

(b) Relay

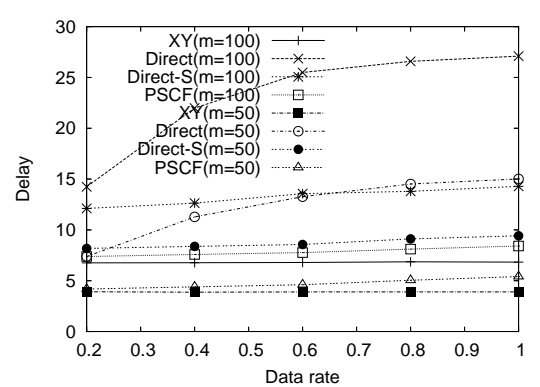

(c) Delay

Fig. 4. Comparison of PSCF, XY, Direct/Direct-S.

with the address $(i, j)$, where $i, j \in 0,1, \ldots, m$. As mentioned in section 3, only nodes in the center $m \times m$ area work as the source/destination. We compare our algorithm, PSCF, with two extremes: (1) X-Y routing (XY) without using mobile nodes, and (2) one-hop approach where the mobile node goes directly to the destination (Direct). Since free-riders are permitted in PSCF, for fair comparison, we also simulate the direct routing with free riders version (Direct-S), where mobile node picks up messages whose destinations are within its trajectory. Thus, even with the free-rider policy, mobile nodes do not change their trajectory, and the numbers of relays of all messages do not change, and the delay of their original messages are not affected.

The following parameters are considered in the simulation: (1) The network size $m$. We use two values, 50 and 100 , to check the scalability of the algorithm. (2) The number of long links $q$. We set $q$ to be 1 in the basic simulation, then we increase it to examine the performance of PSCF with multiple long links. (3) Different data rate, which is represented by data generating probability $p$ of each static node in each round. (4) With and without free-riders. We check the effect of the support of free-riders at intermediate nodes of long links on the overall performance. (5) Different trade points, which is represented by a fixed timer whose value is tunable. (6) With and without reactive long link direction initialization. In the reactive manner, in PSCF, the direction of the long link is initialized based on the destination of the first generated message. (7) With and without trajectory sharing. (8) The density of mobile nodes $d$. We assume that the velocity of mobile nodes is 1 , one time unit, which is also the time unit of the timers. The transmission by short links, including setup time and wireless transmission delay, is assumed to be one time unit or one in a thousand time units when the package is quite small. In the sparse mode, the probability that a grid has a mobile node is $d$.

The performance metrics are:

1) Relative moving distance, which is represented by the ratio between actual moving distance of data and the physical distance of source and destination.

2) The number of relays. This measures the number of hops to transmit message from source to destination.

3) Delay. This is the total time consumption from message source to destination, including waiting and moving time.

4) The delivery ratio. In sparse mode, some generated messages may fail to be delivery due to the lack of mobile nodes.

\section{B. Simulation results}

Figure 4 is the comparison of the four algorithms, $\mathrm{XY}$, Direct, Direct-S, and the proposed PSCF with the basic settings (no free-rider, single long link, no reactive mode). In (a), PSCF has a greater moving distance than the other three, which all deliver data along the shortest path. However, the detour PSCF makes is moderate. The relative moving distance is around 1.015 with $m=100$. In (b) Direct and Direct-S have the smallest fixed number of relays, which is 2. PSCF has a smaller value than XY. (c) shows that Direct has the greatest delay and Direct-S reduces the delay via free-riders, while those of PSCF and $\mathrm{XY}$ are quite small. PSCF has a longer delay than XY because of the waiting done when a long link is chosen. Among all these figures, only the delay of PSCF and Direct/Direct-S vary with the data rate.

Figure 5 is the comparison of PSCF with and without free-riders at the intermediate nodes of long links. Since the support for free-riders provides more available long links, it makes the performance (advantage or disadvantage) of PSCF over XY and Direct more significant. In (a), we can see that with free-riders, PSCF has a larger moving distance. (b) shows that the free-rider policy provides less relays. (c) shows that with free-riders, PSCF has a larger delay.

Figure 6 is the result of the test on trade point in PSCF. We set a fixed timer for each long link. Even if the long link jump condition holds, data is passed on by a short 


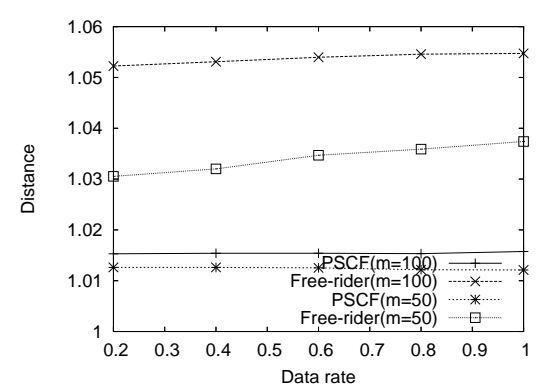

(a) Distance

Fig. 5. PSCF with/without free-riders.

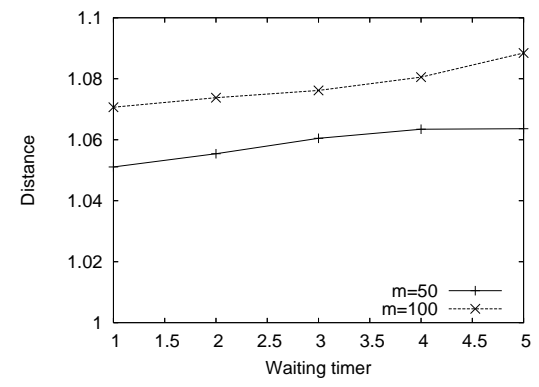

(a) Distance

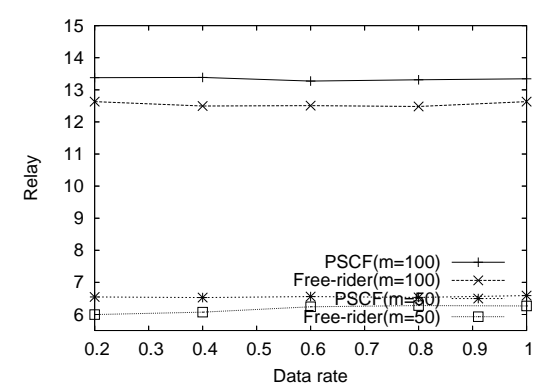

(b) Relay

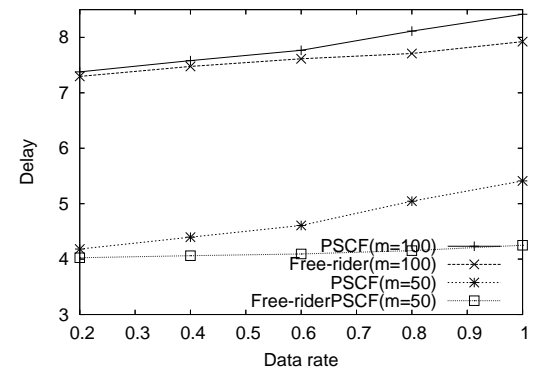

(c) Delay

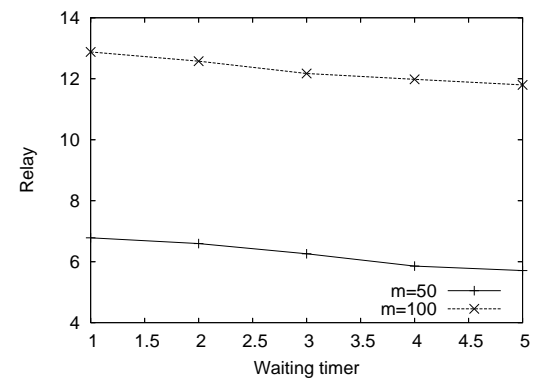

(b) Relay

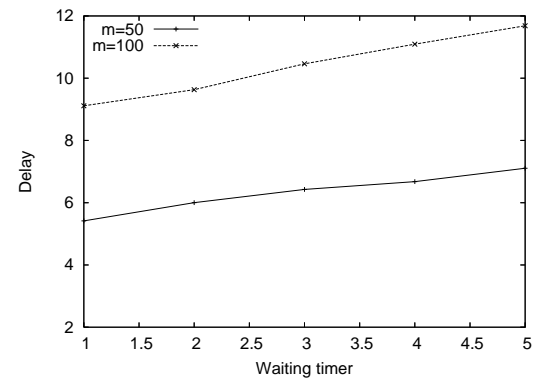

(c) Delay

Fig. 6. PSCF with different timers.

link if the timer expires and the mobile node still does not show up. The longer the allowed waiting time, the larger the moving distance as shown in (a). (b) shows that a longer waiting time makes a fewer number of relays, which makes the long link effectiveness more significant. And (c) shows that longer waiting time also makes PSCF have a larger delay.

Figure 7 shows the performance of PSCF and Direct with multiple long links for each node. PSCF has larger moving distance with more long links as in (a). (b) shows that Direct has fixed number of relays, while PSCF has smaller one when the number of long links is small. (c) shows that the delay of Direct decreases with more available mobile nodes, while that of PSCF increases.

Figure 8 is the comparison of PSCF with or without the reactive long link direction initialization. There are three curves in these figures. One is the basic PSCF, the second is the PSCF with reactive long link initialization (R-PSCF), and the third is the performance of the first generated message in each grid in R-PSCF (R-PSCF-1). In (a), the moving distance of R-PSCF is larger than that of PSCF; in (b), the relay of R-PSCF is smaller than that of PSCF; and in (c), the delay of R-PSCF is larger than that of PSCF. However, since R-PSCF changes only the first message's delivery route, the average performance is seldom affected especially when the simulation lasts a relatively long time. Therefore, the difference between $\mathrm{R}-\mathrm{PSCF}$ and PSCF is slight. R-PSCF-1 is the average performance of the first generated message of each grid. We can see that the performance of R-PSCF-1 is more significant than that of R-PSCF.

Figure 9 (a) is the analysis of long links involving circles in the network, that is, the ratio of long links that form circles. We can see that when the number of long links increases, the ratio increases with it. When the network is smaller, more links are involved in circles. Figure 9 (b) is the delay comparison of PSCF with and without trajectory sharing. With trajectory sharing, the delay decreases slightly. Note that trajectory sharing does not affect moving distance or the number of relays.

Figure 9 (c) shows the comparison of the delivery ratios in the sparse mode. In XY, the sparse mode means that some fixed place holders may missing and their neighbors cannot transmit packets to them. Therefore, the network may get disconnected. In Direct/Direct-S, the sparse mode refers to when mobile nodes are missing in the place holders as in the PSCF method. The delivery ratio of Direct is approximate the density of mobile nodes. This is due to the fact that the generated message can only be delivered if there is a mobile node in the grid. The delivery ratio of Direct-S is slightly higher than that of Direct, since with the help of sharing, some grids without 


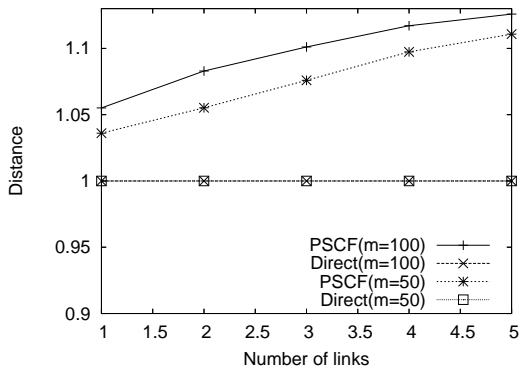

(a) Distance

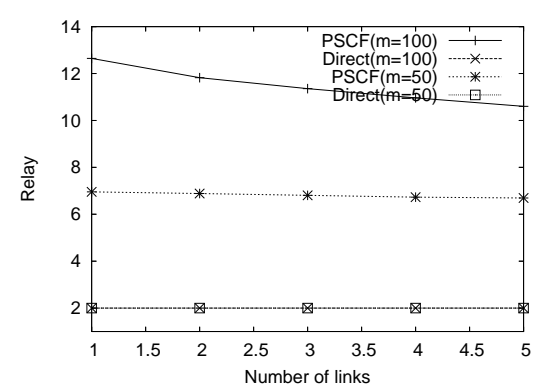

(b) Relay

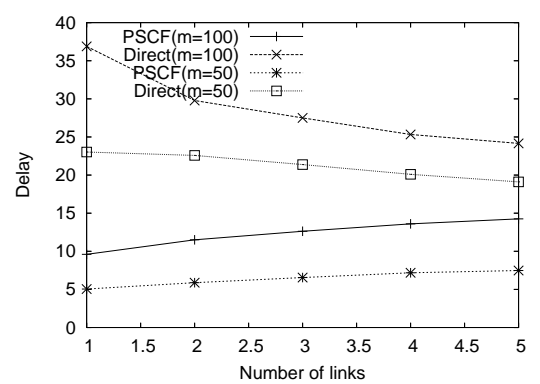

(c) Delay

Fig. 7. PSCF with multiple long links.

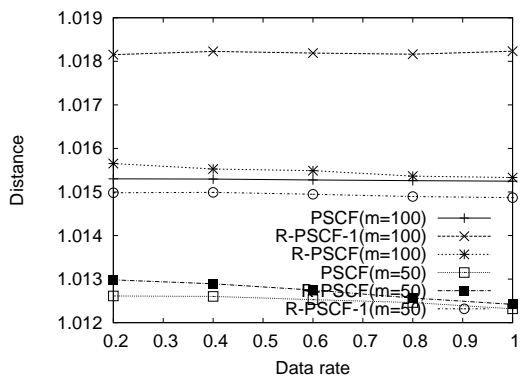

(a) Distance

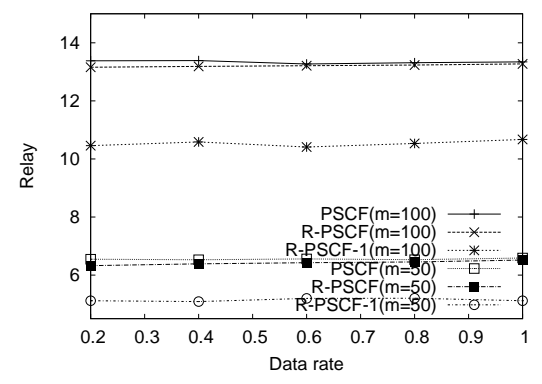

(b) Relay

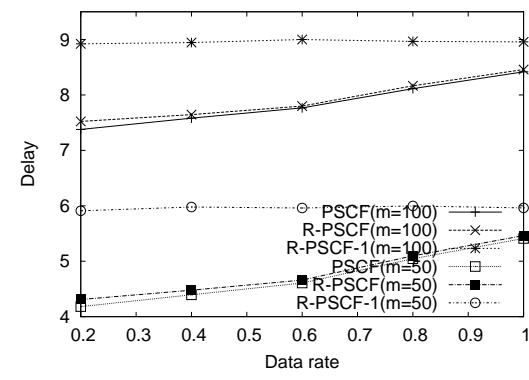

(c) Delay

Fig. 8. PSCF with/without reactive long link.

mobile node can also send out the generated message via by-passing mobile nodes. When the density of the mobile nodes is small, XY has a smaller delivery ratio than that of Direct due to the high degree of disconnectivity. When the density $d$ exceeds 0.5 , the delivery ratio of $\mathrm{XY}$ increases quickly and outperforms that of Direct. PSCF has the largest delivery ratio.

Figure 9 (d) shows the delay comparison of PSCF, XY, Direct, and Direct-S when the time consumption of one hop wireless transmission is of one in a thousand time units. We can see that in this case, the delay of $\mathrm{XY}$ is very small compared with the other methods. The delay of PSCF is smaller than that in Figure 4 (c) since it also contains some short link transmission in the entire trajectory. Although the delay of PSCF is significantly higher than that of $\mathrm{XY}$, it reduces the number of relays, and more important, increases the delivery ratio in sparse network, as in Figure 9 (c).

The simulation results can be summarized as follows:

1) The proposed algorithm generates a smaller number of relays than $X Y$ while still maintaining a moderate moving distance and delay which makes PSCF more suitable for wireless ad hoc or sensor networks.

2) When we analyze the properties of PSCF, we can see that a larger network makes the performance of PSCF more significant, and also the support for free-riders, multiple long links, longer waiting time for the long links, and reactive long link initialization.

3) Trajectory sharing can improve the performance of PSCF in terms of delay slightly, while not affect moving distance or relay.

4) In the sparse mode, PSCF has the largest message delivery ratio compared to $\mathrm{XY}$ and Direct, especially when the network is relatively sparse.

\section{CONCLUSIONS}

In this paper, we propose a new controlled mobility model with an expected polylogarithmic average number of relays to achieve a good balance among several contradictory goals. The model is based on the small-world model where each node has "short" link connections to its nearest neighbors and "long" link connections to other nodes following a certain probability distribution. Several dynamic trajectory planning and sharing methods for mobile nodes are proposed to enhance the efficiency. In our future work, we will include more analytical study, including network throughput analysis. More in-depth simulation and parameter trade-offs will also be studied. 


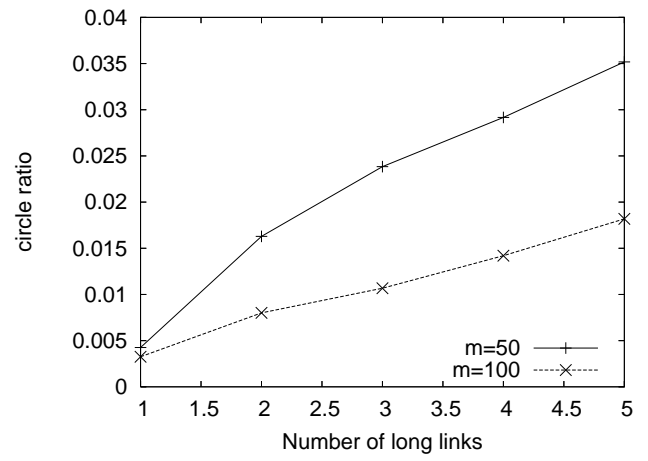

(a)

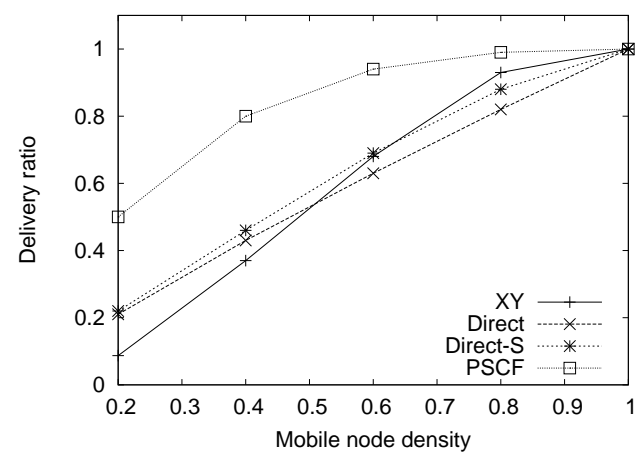

(c)

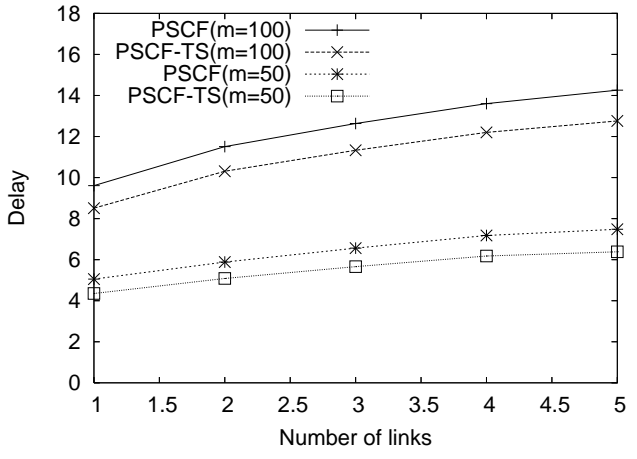

(b)

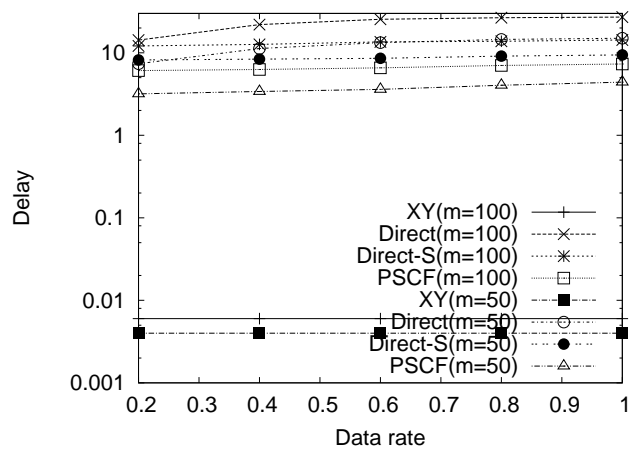

(d)

Fig. 9. (a) Percentage of circle involved long links, (b) delay of PSCF with and without trajectory sharing, (c) delivery ratio in sparse mode, and (d) delay comparison when wireless transmission is of one in a thousand time unit.

\section{ACKNOWLEDGMENTS}

This work was supported in part by NSF grants ANI 0083836, CCR 0329741, CNS 0422762, CNS 0434533, EIA 0130806, CNS 0531410, and CNS 0626240.

\section{REFERENCES}

[1] L. Blazevic, L. Buttyan, S. Capkun, S. Giordano, J. Hubaux, and J. Le Boudec. Self-organization in mobile ad-hoc networks: the approach of terminodes. IEEE Communications Magazine, 2001.

[2] L. Bodin, B. Golden, A. Assad, and M. Ball. Routing and scheduling of vehicles and crews - the state of the art. Computers and Operations Research, 10:63C211, 1983.

[3] R. Chitradurga and A. Helmy. Analysis of wired short cuts in wireless sensor networks. In Proc. of IEEE International Conference on Pervasive Svcs., 2004.

[4] N. Christofides, A. Mingozzi, and P. Toth. Exact algorithms for the vehicle routing problem, based on spanning tree and shortest path relaxations. Mathematical Programming, (1):255-282, 1981.

[5] S. Das, A. Nandan, G. Pau, M.Y. Sanadidi, and M. Gerla. Evaluating mobility pattern space routing for DTNs. In Proc. of the 1st ACM VANET, 2004.

[6] M. Grossglauser and D. Tse. Mobility increases the capacity of ad-hoc wireless networks. In Proc. of IEEE INFOCOM, 2001

[7] P. Gupta and P. R. Kumar. The capacity of wireless networks. IEEE Transactions on Information Theory, 46(2):388-404, 2000.

[8] B. Hull, V. Bychkovskiy, K. Chen, M. Goraczko, E. Shih, Y. Zhang, H. Balakrishnan, and S. Madden. CarTel: A distributed mobile sensor computing system. In Proc. of the SenSys, 2006.
[9] J. Kleinberg. The Small-world phenomenon: An algorithmic perspective. In Proc. of the 32nd ACM Symposium on Theory of Computing, 2000.

[10] J. Kleinberg. Small-world phenomena and the dynamics of information. Advances in Neural Information Processing Systems (NIPS) 14, 2001

[11] T. Small and Z. Haas. The shared wireless infostation model a new ad hoc networking paradigm (or where there is a whale, there is a way). In Proc. of ACM MobiHoc, 2003.

[12] T. Dimtar et al. Small world application layer for ad hoc networks. Telekomunikacioni Forum Telfor, Belgrade, 2003.

[13] A. Vahdat and D. Becker. Epidemic routing for partially-connected ad hoc networks. In Technical report, Duke University, 2002.

[14] W. Wang, V. Srinivasan, and K. C. Chua. Using mobile relays to prolong the lifetime of wireless sensor networks. In Proc. of ACM MobiCom, 2005.

[15] D. Watts and S. Strogatz. Collective dynamics of small-world networks. Nature 393, 440 (1998).

[16] V. W.-S. Wong and V. C.M. Leung. Location management for next-generation personal communicaiton networks. IEEE Networks.

[17] J. Wu and F. Dai. Mobility-sensitive topology control in mobile ad hoc networks. In Proc. of IEEE IPDPS, 2004.

[18] Y. Xu, J. Heidemann, and D. Estrin. Geography informed energy conservation for ad hoc routing. In ACM/IEEE International conference on Mobile Computing and Networking, 2001.

[19] J. Zhao and G. Cao. VADD: Vehicle-assisted data delivery in vehicular ad hoc networks. In Proc. of the IEEE INFOCOM, 2006.

[20] W. Zhao, M. Ammar, and E. Zegura. Controlling the mobility of multiple data transport ferries in a delay-tolerant network. In Proc. of IEEE INFOCOM, 2005. 